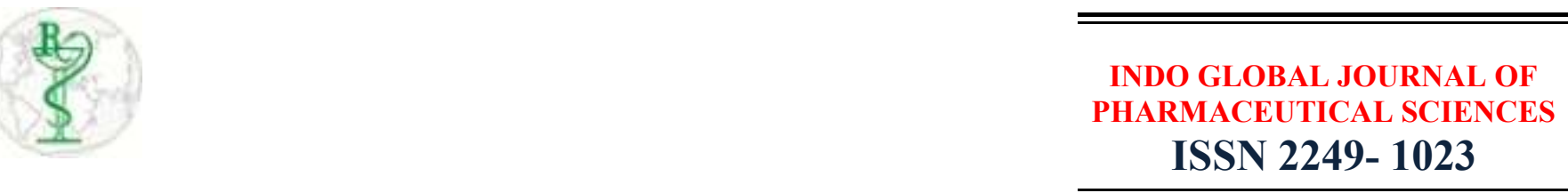

\title{
Impedimetric Immunosensor for Prostate Specific Antigen
}

\author{
Rahul Saxena, Ghandharav Thakur, Deepshikha Tyagi, Madhur Arya, Shikhar Bhola, Nidhi \\ Bhandari, Sudha Srivastava *
}

Department of Bioetchnology, Jaypee Institute of Information Technology, Sec 62, Noida, India

Address for Correspondence: Sudha Srivastava, sudha.srivastava@jiit.ac.in

Keywords PSA; Immunosensor; Biomarker.
ABSTRACT: We report an impedimetric biosensor for quantification of Prostate-specific antigen (PSA) in sexual assault cases. PSA, Acid Phosphatase and Semenogelin are the three biomarkers employed for detection of semen in samples. Short life span of semenogelin and reduction in enzymatic activity of acid Phosphatase leaves PSA as preferred biomarker of choice for forensic investigations confirming the presence of seminal fluid in sexual assault cases. We have developed an immunosensor based on direct ELISA format by immobilizing anti-PSA antibody onto nanoparticles modified electrode surface. Quantification of PSA in simulated semen samples using the developed immunosensor displayed a lower detection limit of 0.001 $\mathrm{ng} / \mathrm{mL}$ as opposed to $4 \mathrm{ng} / \mathrm{mL}$ of that of ELISA. (C) 2016 iGlobal Research and Publishing Foundation. All rights reserved.

Conference Proceedings: International Conference on Advances in Plant and Microbial Biotechnology (PMB2017); JIIT, Noida: February 02-04, 2017

Indo Global Journal of Pharmaceutical Sciences( ISSN 22491023 ; CODEN- IGJPAI; NLM ID: 101610675) indexed and abstracted in EMBASE(Elsevier), SCIRUS(Elsevier),CABI, CAB Abstracts, Chemical Abstract Services(CAS), American Chemical Society(ACS), Index Copernicus, EBSCO, DOAJ, Google Scholar and many more. For further details, visit http://iglobaljournal.com 